\title{
Enhancing Everyday Paper Interactions with Paper Circuits
}

\author{
Michael Shorter \\ Eclectric Research Studio \\ University of Dundee \\ mail@mrshorter.co.uk
}

\author{
Professor Jon Rogers \\ Eclectric Research Studio \\ University of Dundee \\ j.rogers@dundee.ac.uk
}

\author{
Dr John McGhee \\ 3D Visualisation Aesthetics Lab \\ University of New South Wales \\ John.mcghee@unsw.edu.au
}

\begin{abstract}
Our interactions with paper are so habitual as to be subconscious. Paper is an inextricable component of our daily lives. In this paper we present the crafting of, and the reflections on, four prototypes; these prototypes explore how adding new functionality through paper circuits can enrich interactions with paper. We define paper circuits as circuits that have been made through the process of printing or applying conductive ink onto standard paper in order to form electronic or electric circuits. We will provide reflections on not just the benefits of paper circuitry, but also how the newly added affordances gained from paper circuitry effect the experience of paper interactions. This paper will illustrate how this new evolution of paper can be used to produce cheap lightweight ubiquitous electronic products, new art forms, and most importantly enhance the user experience of paper without losing the existing wellloved affordances that paper currently possesses.
\end{abstract}

\section{Author Keywords}

Design; Paper; Electronics; Circuit; Prototyping.

\section{ACM Classification Keywords H.5.0 General.}

\section{INTRODUCTION}

In the book Pulp: Paper Jon Cefai adeptly explains why we have such an emotional connection to paper:

"Paper can be memorable, beautiful and have a keepsake value that makes you want to collect it. Its permanence and its fragility, two colliding properties that can make it something you want to protect... Paper's ability to display allows us to surround ourselves with memories. It can colour our lives and become a part of our identity."[7]

Permission to make digital or hard copies of part or all of this work for personal or classroom use is granted without fee provided that copies are not made or distributed for profit or commercial advantage and that copies bear this notice and the full citation on the first page. Copyrights for thirdparty components of this work must be honored. For all other uses, contact the Owner/Author.

Copyright is held by the owner/author(s).

DIS '14, Jun 21-25 2014, Vancouver, BC, Canada

ACM 978-1-4503-2902-6/14/06.

http://dx.doi.org/10.1145/2598510.2598584
What would happen if physical paper became smarter, or acquired added behaviours and functionality?

The HCI field has always explored new technologies that aim to replace paper. Display Stacks and Paper Tab by the Human Media Lab, Bookisheet by Watanabe et al. and PaperPhone by Lahey et al. are all prototypes that mimic the interactions we have with paper without actually using paper $[5,16,17,8]$. There are also examples of work where paper is augmented using hardware such as computers and projectors. Examples of this are Listen Reader by Back et al., PapierCraft by Liao et al. and Flexpad by Steimle et al. $[1,9,15]$. While the aforementioned examples are valuable for exploring potential futures for paper, there are inherent compromises. Not only do they lose that emotionally important 'papery' feel, they also require external hardware, which makes them difficult to take into the field. These studies are looking for something to replace paper, however, studies like Sellen and Harper's The Myth of the Paperless Office have proved that there are some qualities of paper we just can't let go of [12], Consider the fact that global paper consumption has doubled since the publication of The Myth of the Paperless Office, despite technological advancements. Paper circuits don't try to change paper's natural affordances, but work alongside them. Paper circuitry is honest; there are no computers hidden under tables, or projectors behind doors. Everything is physically included in the prototype, and paper circuits shy away from unnecessary complexity and expense. Paper circuitry's recent ability to give standard paper digital interactivity identifies it as the next step in the field of HCI.

All of the previous examples have shown how paper affordances can be brought to electronics [10]. In this paper we explore how electronic affordances can be combined with paper using paper circuits.

Paper circuitry is the printing (or painting) of conductive ink on standard paper. There are a range of different conductive inks and paints; carbon-based, silver-based, copper-based, graphene-based, nickel-based and silver nanoparticle-based to name a few. When printed on paper, conductive ink can be used to create basic components such as resistors, capacitors, speakers, microphones and even transistors. It is also very easy to transform paper into touch screens using capacitive touch. Complex paper circuits can be created by connecting traditional components to paper 
using conductive inks and epoxies. These inks can be applied to the paper substrate using different traditional techniques including silkscreen printing, flexography, gravure printing, offset lithography, inkjet printing, and painting. By approaching an emerging technology such as paper circuitry with these traditional craft techniques we are able to prototype effectively as traditional craft techniques are inherently reflective [14].

There is some relevant existing work exploring paper circuits. Pulp-Based Computing by Coelho et al., Paints, Paper and Programs by Buechley et al. and Instant Inkjet Circuits by Kawahara et al. all explore creating selfcontained circuits on paper substrates [4, 2, 6]. These examples, however, only explore techniques and basic functions rather than designed applications for specific contexts. It is worth mentioning that there are various companies starting to produce both paper circuits and platforms for paper circuits on a commercial level.

\section{PROTOTYPES (RESEARCH IMPERITIVES)}

We built and tested a series of prototypes in order to demonstrate how paper's existing affordances can be enhanced using paper circuits. These four prototypes each demonstrate a different paper-based interaction. For each example there is an explanation of what the affordances are, what the prototype does, how it was crafted and reflections on how the affordances benefit from paper circuitry. Each prototype's evaluation will use reflective practice, the authors' methodology of choice, to draw insights relevant to the crafting of paper experiences [13].

\section{The Invite}

Paper is often used as a call to action. Invitations, for example, are mailed out, taken to events and exchanged for experiences. They can be beautiful things with well-crafted affordances; graphic design, size, paper stock and content are all considered. A physical invitation can be more powerful than an e-invitation, or 'e-vite' [7]. We questioned what would happen if the physical invitation's careful aesthetic considerations were combined with paper circuits and our answer was the Invite.

The Invite was screen printed with carbon-based conductive ink. It contained no hardware, which made it look just like a normal printed piece of paper. However, when plugged into the speaker system at an event, the Invite became a musical instrument (Figure 1). There were no added costs of electronic components per flyer; the main cost was in the base unit that the Invite plugged into.

The Invite interface allowed control of audio output in two different ways: pitch and frequency. The large circle used capacitive sensing to create what acted like a distance sensor. The distance your hand was from the printed area determined the pitch of the sound. The three smaller circles controlled three frequency settings. This was achieved by also using capacitance, but this time as touch buttons. The

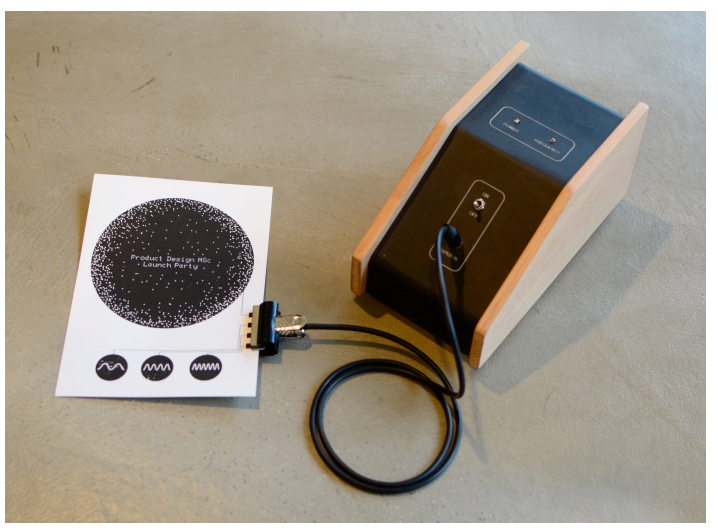

Figure 1. The Invite plugged into the base unit.

base unit that the Invite plugged into contained all the hardware. This unit used an Arduino to sense what part of the paper had been touched. A bespoke bulldog clip was created to connect the Invite to the base unit.

Adding paper circuitry to an invitation can result in increased curiosity, examination and play - important outcomes to consider when adding levels of interactivity and physicality to a piece of print. With the addition of paper circuitry, the Invite gained intriguing new affordances that, despite their newness, did not detract from the traditional affordances of paper. For very little extra cost the experience of an invitation was enhanced; the Invite now afforded a musical interface. Creating the Invite with paper circuits meant that it could still adhere to existing social interactions such us mailing, carrying around in a pocket, and being used at an event.

\section{Asteroids}

Every bedroom, school and university has educational posters on the walls. These posters currently afford the distribution of data. What would happen if these posters could display meaningful, dynamic data rather than static data?

Asteroids is an A3 Internet-connected poster that visually illustrates the size and proximity of asteroids passing earth (see Figure 2). This prototype was influenced by existing paper star charts and designed to fit within this context. Asteroids was constructed by laser cutting electroluminescent paper into asteroid shapes and sticking them to the back of the standard paper substrate so when illuminated, they shone through. The trajectory of the asteroids was depicted through lines of surface mount LEDS attached to the back that again, shone through when illuminated. The data was retrieved from the Internet using a small wireless Internet-enabled SD card called an Electric Imp. The Electric Imp fed the data to an Arduino Nano, which did the computing to control various asteroid sizes and trajectories via a set of surface mount relays. Asteroids needed to be powered using a $9 \mathrm{~V}$ battery due to the high power consumption of the electroluminescent paper. The components were connected using silver conductive ink. 


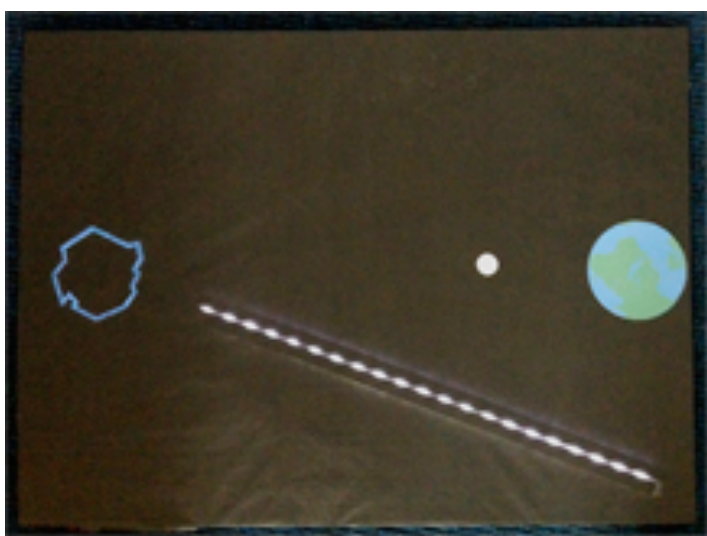

Figure 2. The Asteroids displaying data.

The ubiquity of posters makes this application of paper circuitry appropriate. There is an existing infrastructure in educational institutions for posters that paper circuitry posters would not disrupt. Screens are the only option for delivering the same amount of interactive information as a paper circuitry poster, but they also hold pitfalls of being potentially overwhelming, and expensive. Asteroids illustrated that an inexpensive paper circuit poster could deliver complex data in a simple yet stimulating manner. The potential for a paper circuitry poster to increase the distribution of knowledge is significant. By joining paper circuits with the Internet of Things movement we are able to give people access to more information than a normal printed poster can provide [18].

\section{Paper Headphones}

Paper affords customisation, whether through folding, cutting or graphics. Paper craft and origami has always been a popular pastime. What would happen if these craft practices were combined with paper circuits to add extra interactivity?

The Paper Headphones were designed and created in collaboration with Novalia as a promotional give-away for the Can Printed Electronics Save the Music Industry? panel talk in the Interactive section at SXSW 2012 [11, 3]. The headphones were presented in an A2 poster format; they could be popped out and stuck together to create a fully working pair of paper headphones (Figure 3). The Paper Headphones were printed using offset lithography with silver conductive ink to create a simple circuit. Around 150 headphones were printed in total. Perforations were then laser cut into each pair. One-inch $(25 \mathrm{~mm})$ piezo speakers were then stuck onto the paper and connected to the conductive ink using conductive epoxy. Finally, a headphone cable was soldered onto each pair. When plugged into an audio device, the Paper Headphones worked just like normal headphones.

Printing on paper resulted in lightweight, low cost, and easily transportable headphones. The recipients of the Paper Headphones were all very excited to build them and they

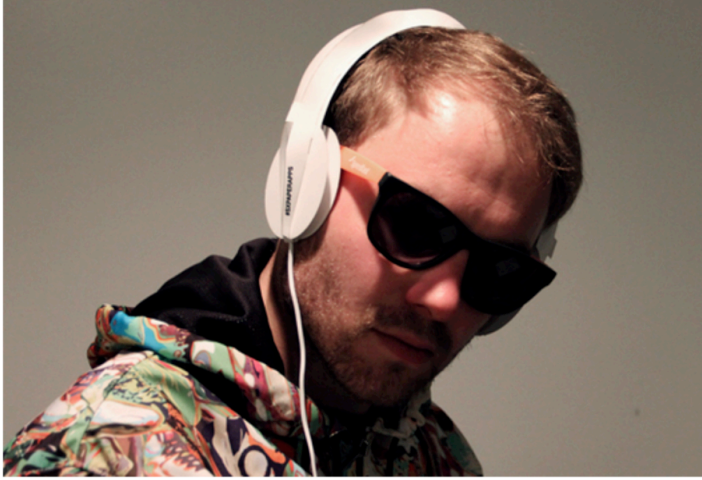

Figure 3. The Paper Headphones in action.

worked effectively as an advertising tool despite their indisputable novelty value. The Paper Headphones are a pertinent example of affordances changing when paper circuits are added; cheap paper structures suddenly have added value. The motivation for this prototype was to test the potential to batch produce paper circuitry.

\section{HD Ready: A Simple Robot}

An artist has a physical relationship with paper that could never be replicated with screen-based art. The affordances and preconception of value of a piece of paper completely change the minute it is put in a frame. What would happen if framed paper artworks were given added behaviours using paper circuitry? How would this affect not only the affordances of the art, but also the relationships between the artist, the artwork and the viewer?

HD Ready: A Simple Robot is a paper-based artwork from fine artist Jack Paton (Figure 4). The platform allowing the artwork to be connected and printed was developed by the

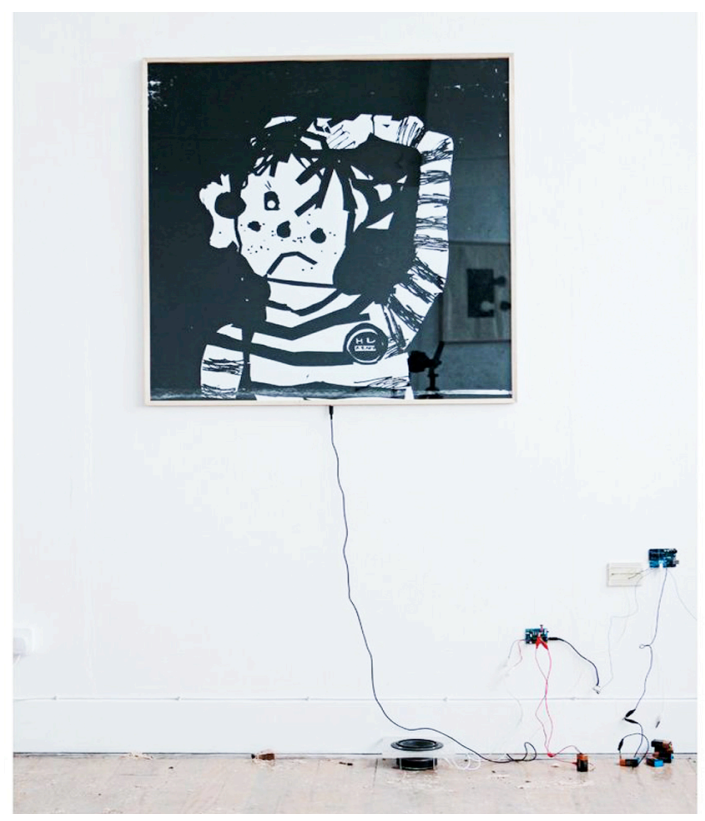

Figure 4. HD Ready: A Simple Robot in a gallery setting. 
author of this paper. The artwork was screen printed with carbon-based conductive ink and connected to an Arduino that controlled a loudspeaker. The pitch of the audio was controlled by both the amount of people in the gallery and their proximity to the artwork. The artwork was grounded by a cable connecting it to a water pipe. When a person viewed the artwork they unwillingly entered a relationship with not only the artwork, but also the entire building's plumbing and electrical system.

Combining art with paper circuitry not only gave the artist more creative options for a familiar medium but also enhanced the gallery experience for viewers by giving them the unusual opportunity to not only connect with, but also affect, the artwork. HD Ready: A Simple Robot also tested large area screen printing with carbon-based conductive ink $\left(1 \mathrm{~m}^{2}\right)$ and challenged notions of what a circuit looks like, as the circuit itself was part of the artwork.

\section{CONCLUSION}

Exploring these four prototypes has demonstrated that applying craft methodology is both useful and appropriate when enhancing paper interactions with paper circuitry. In this paper we detail techniques and methods used to not only print with conductive ink, but also attach components and connect paper circuits to external devices. Each prototype illustrates how paper circuitry can enhance paper's affordances without compromising the traditional experience. We found that exploring new ways to work and interact with such a familiar medium fostered creativity revealed potential and ultimately bolstered paper circuitry's position as a valuable emerging field.

The new knowledge contributed to the field of paper circuitry through this study has come through traditional craft approaches to prototyping and includes, but is not limited to, discoveries around creating live dynamic data displays, the potential mass production of inexpensive, recyclable and lightweight products and the challenging of preexisting notions of circuitry through the honest and obvious integration of circuits in graphic art. Introducing fresh affordances creates a spike of interest that revitalises paper and its traditional affordances that we have grown so accustomed to. Paper is, after all, both a product and a tool of craft, and deserves to have its rich history and traditions take it forward towards exploring new applications and relevancies in the digital age.

\section{ACKNOWLEDGMENTS}

We thank Novalia and the EPSRC for funding this research.

\section{REFERENCES}

1. Back, M., Cohen, J., Gold, R., Harrison, S. and Minneman, S. Listen reader: an electronically augmented paper-based book. Proc. CHI 2001, ACM (2001), 23-29.
2. Buechley, L., Hendrix, S. and Eisenberg, M. Paints, paper, and programs: first steps toward the computational sketchbook. Proc. TEI 2009, ACM (2009), 9-12.

3. Can Printed Electronics Save the Music Industry?, 2012. SXSW Schedule 2012. URL http://schedule.sxsw.com/ 2012/events/event_IAP11099

4. Coelho, M., Hall, L., Berzowska, J. and Maes, P. Pulpbased computing: a framework for building computers out of paper. Ext. Abstracts CHI 2009, ACM (2009), 3527-3528.

5. Girouard, A., Tarun, A. and Vertegaal, R. DisplayStacks: interaction techniques for stacks of flexible thin-film displays. Proc. CHI 2012, ACM (2012), 2431-2440.

6. Kawahara, Y., Hodges, S., Cook, B.S., Zhang, C. and Abowd, G.D. Instant inkjet circuits: lab-based inkjet printing to support rapid prototyping of ubicomp devices. Proc. UbiComp 2013, ACM (2013), 363-372.

7. KentLyons, 2010. Pulp-Paper.

8. Lahey, B., Girouard, A., Burleson, W. and Vertegaal, R. PaperPhone: understanding the use of bend gestures in mobile devices with flexible electronic paper displays. Proc. CHI 2001, ACM (2001), 1303-1312.

9. Liao, C., Guimbretire, F. and Hinckley, K. PapierCraft: a command system for interactive paper. Proc. UIST 2005, ACM (2005), 241-244.

10.Norman, D.A., 2002. The Design of Everyday Things. Basic Books, New York.

11. Novalia, 2012. URL. http://novalia.co.uk/

12. Sellen, A.J. and Harper, R. The Myth of the Paperless Office. MIT Press, Cambridge, MA, 2002.

13. Schön, D.A., 2003. The reflective practitioner : how professionals think in action. Ashgate, Aldershot.

14. Shorter, M., Rogers, J., Metcalfe, T., McGhee, J., 2013. The invite: adding value to paper with paper electronics. Proc. Research Through Design 2013, pp. 35-38.

15. Steimle, J., Jordt, A. and Maes, P. Flexpad: highly flexible bending interactions for projected handheld displays. Proc. CHI 2013, ACM (2013). 237-246.

16. Tarun, A., Wang, P., Strohmeier, P., Girouard, A., Reilly, D. and Vertegaal, R. PaperTab: tablets as thin and flexible as paper. Ext. Abstracts CHI 2013, ACM (2013), 2881-2882.

17. Watanabe, J., Mochizuki, A. and Horry, Y. Bookisheet: bendable device for browsing content using the metaphor of leafing through the pages. Proc. UbiComp 2008, ACM (2008), 360-369.

18. Webb, M., 2013. Berg Cloud. URL http://www.bergcloud.com 